\title{
THICKNESS DEPENDENCE OF EFFECTIVE CRITICAL EXPONENTS IN THREE-DIMENSIONAL ISING PLATES
}

\author{
M.I. MARquÉS AND J.A. Gonzalo*
}

Departamento de Física de Materiales, C-IV

Universidad Autónoma de Madrid, 28049 Madrid, Spain

(Received October 20, 1999; revised version January 17, 2000)

Phase transitions in Ising plates of equal area and different thicknesses have been studied by the Monte Carlo approach. The evolution of the critical temperature and of the effective critical exponents with the thickness of the lattice has been numerically determined. The thickness dependence of the maximum value of the effective critical exponents is well described by an exponential decay towards the respective three-dimensional value.

PACS numbers: $75.10 . \mathrm{Hk}, 64.60 . \mathrm{Fr}, 75.70 .-\mathrm{i}, 02.50 . \mathrm{Ng}$

\section{Introduction}

Phase transitions in systems with a thin film geometry have been extensively studied both experimentally and theoretically in recent years. Research has been focused mainly in the following two points:

First, surface effects in thin films have been studied by several authors $[1,2]$. Special attention has been paid to the possibility that the interaction constant at the surface becomes different from that at the bulk. These authors find that the critical exponents for the surface are different from those for the bulk. Scaling, multicritical points, and crossover have also been studied as the surface interaction changes with respect to the bulk interaction [1].

Second, the evolution of the effective critical exponents with temperature in the close vicinity of $T_{\mathrm{c}}$ for systems with the thin film geometry and with free surfaces has been studied by means of series expansion [3], the renormalization group [4] and high accuracy Monte Carlo calculations in Ising systems [5] and in the $\mathrm{X}-\mathrm{Y}$ model [6]. Attention was given to the study of the crossover phenomena in the effective critical exponents from three-dimensional to two-dimensional as the system approaches the critical temperature.

*e-mail: julio.gonzalo@uam.es 
For a thin film Ising system with infinite surface, the correlation length, sufficiently below and above $T_{c}$, is much smaller than the film thickness, and the behavior of the system tends to be the one corresponding to a three-dimensional one, but as it approaches the critical point, the correlation length becomes bigger than the thickness, and the system shows the crossover of the effective critical exponents towards two-dimensional critical exponents. This implies that a thin film of infinite surface belongs necessarily to the two-dimensional universality class.

So, basically, if we focus our attention on the critical behavior, thin films cannot be distinguished from two-dimensional systems, since their thickness is much smaller than their surface dimensions. However, the behavior of the effective exponents departs strongly from the one corresponding to the two-dimensional or the three-dimensional systems, and, furthermore, it is characteristic of the particular thickness of the system under consideration. Our aim in this work will be to find characteristic effective critical exponents capable of characterizing the thickness of the system under study.

Real thin films grown on top of substrates may be substantially constrained at least at one of the surfaces even if the other surface is free. Therefore, the appropriate boundary conditions may be intermediate between free and constrained (periodic).

\section{Numerical results}

In this work we will use numerical finite-size lattice Monte Carlo simulations of phase transitions in Ising systems with periodic boundary conditions for different thicknesses, in an effort to determine the dependence of the effective critical exponents on the thickness of the system at the transition from two-dimensional to three-dimensional. Periodic boundary conditions will be considered in every direction as a way to by pass surface effects. A mixture of Metropolis/Wolff single cluster Monte Carlo calculations [7] for lattices with a constant linear dimension $(L=100)$ and different thicknesses $(D=3,5,7)$ were performed. Our Monte Carlo calculations involve about 50000 Monte Carlo steps per spin for each data point, which is needed to get good enough statistics. Initial conditions for each value were taken as the equilibrium conditions for the previous temperature. The calculations were performed at temperatures near to zero (or strong fields) in order to ensure equilibrium. We use a random number generator as previously given in Ref. [8].

In order to study the evolution of the effective critical exponents at the transition from film to bulk, it is of course necessary to study first the evolution of the critical temperature with the thickness of the system. The work of this kind was done some time ago [9] and also more recently by Schilbe et al. [5]. The critical temperatures for the different film thicknesses investigated were calculated using the Binder-cumulant $U_{L}$ method [10] for different lateral sizes of the films. Results for the critical temperature are shown in Fig. 1a for $D=1,3,5,7$. The scaling prediction $[11,12]$

$$
1-\frac{T_{\mathrm{c}}(D)}{T_{\mathrm{c}}{ }^{3 D}} \propto D^{-\lambda}
$$


where $\lambda=1 / \nu_{3 D}$, holds in our data for all the values $D$ considered. This is at variance with the result in Ref. [5], where Eq. (1) is fulfilled only for thicknesses larger than 20 layers. This is due to the use of periodic boundary conditions in our case. Surface effects introduced by the application of free boundary conditions affect the value of the measured critical temperature and may make Eq. (1) inapplicable for small enough thicknesses $(D<20)$. To check this we have done a numerical calculation of the critical temperature of an Ising system with $D=3$ and free boundary conditions at the surface. The result is shown in Fig. 1b, in comparison with the result for the periodic boundary condition. Now we find a value for the critical temperature very close to the one reported in Refs. [5, 9].
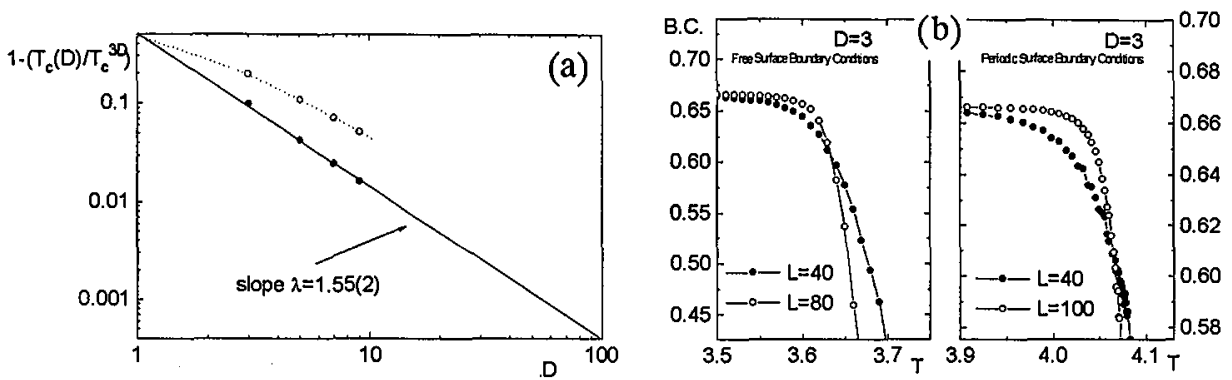

Fig. 1. (a) Dependence of the critical temperature on the thickness for periodic (black) and free (white) boundary conditions. Note that data for periodic boundary conditions fit well the expression given in Eq. (1). (b) Binder cumulant vs. temperature for Ising systems with $D=3$. Note the difference on $T_{\mathrm{c}}(D)$ between the free surface case and the periodic case.

The Binder-cumulant value found for the critical thickness corresponding to the two-dimensional case was, as expected, in very good agreement with the value given previously in the literature [13]. $U_{L}$ is also well known for the three-dimensional systems [14] and it was confirmed with our results for $L^{3}$ systems.

Once we know the critical temperatures for each $D$ we calculate first the effective critical exponent delta $(\delta)$ defined as

$$
\delta=\left.\frac{\partial \log H}{\partial \log m}\right|_{t \rightarrow 0} .
$$

Here $m$ is the magnetization per spin, $H$ is the applied magnetic field and $t$ is the temperature difference relative to $T_{\mathrm{c}}$ for each $D$, i.e. $t=\left|T_{\mathrm{c}}(D)-T\right|$.

To calculate the effective critical exponent $\delta$ we have performed Monte Carlo simulations of the magnetization with different applied fields at $T=T_{\mathrm{c}}(D)$. Results for $\log m$ vs. $\log H$ are shown in Fig. 2a.

This calculation clearly shows two effects. At large $\log H$, the system is close to saturation, moving away from saturation the inverse effective critical exponent (in this case $1 / \delta$ ) must increase. The opposite should happen when we begin to get closer to the critical point $(m=0, H=0)$. Then the crossover to the two-dimensional behavior acts so as to decrease the value of the slope in Fig. 2a. The idea is that if we represent $1 / \delta$ vs. $\log H$ we must find a maximum 

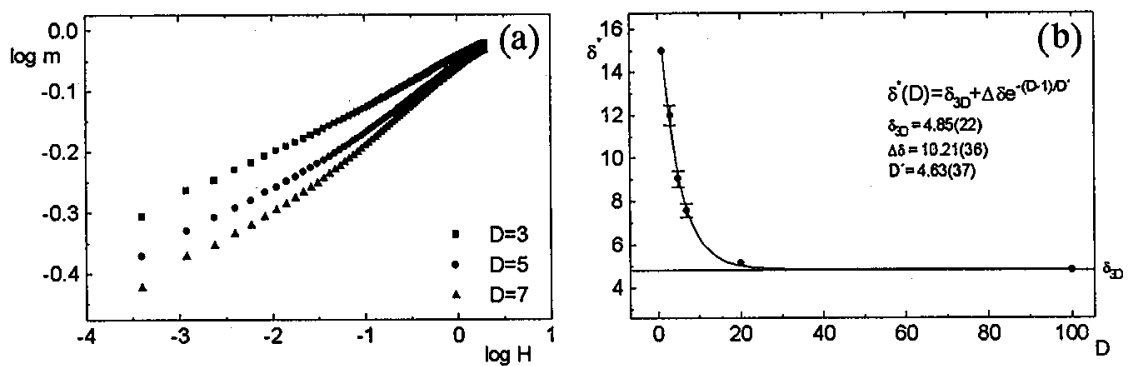

Fig. 2. (a) Log of the magnetization per spin versus log of the applied magnetic field for different thicknesses and for a value of $L=100$. (b) Dependence of the critical exponent $\delta^{*}$ on thickness. The data follow an exponential decay ( $D=20$ from Ref. [5]).

value [15], just before the crossover starts. We call this maximum value $1 / \delta^{*}(D)$, which indicates explicitly the $D$ dependence.

So for all the cases corresponding to different $D$ values we can estimate the maximum of $1 / \delta$. Figure $2 \mathrm{~b}$ shows the values $\delta^{*}(D)$ obtained this way for various thicknesses. Note that $\delta^{*}(D)$ is characteristic of each $D$, and how the three-dimensional value is approached at relatively small values of the thickness. In particular, we find an exponential decay towards this three-dimensional value, as shown in Fig. $2 \mathrm{~b}$.

Next is the calculation of the effective critical exponent beta $(\beta)$ defined as

$$
\beta=\left.\frac{\partial \log m}{\partial \log t}\right|_{H \rightarrow 0} \text {. }
$$

Again, to calculate this critical exponent we have performed Monte Carlo simulations of the magnetization for different temperatures when $H=0$ (no external filed applied). Results for $\log m$ vs. $\log t$ thus obtained are shown in Fig. 3a.

We find the same two effects as before, now temperature playing the role previously played by magnetic filed. Again, we find a maximum value for the
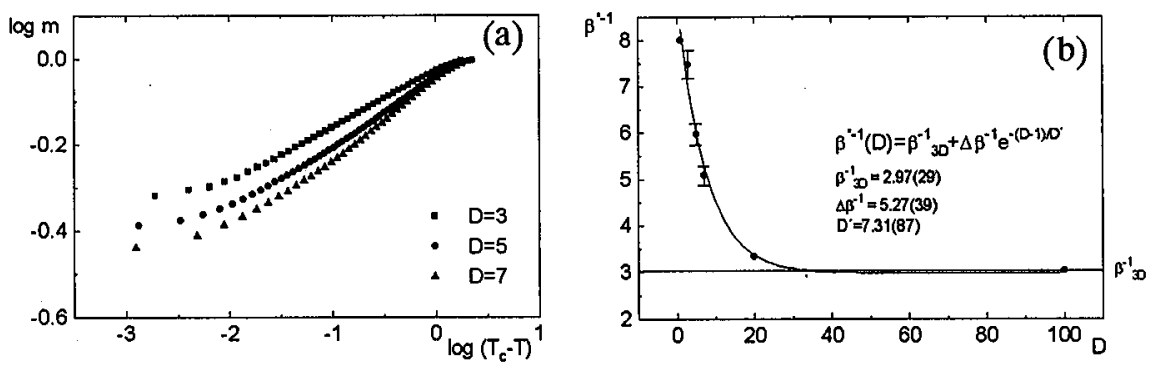

Fig. 3. (a) Log of the magnetization per spin versus log of the difference in temperature relative to $T_{\mathrm{c}}\left(T_{\mathrm{c}}(D)-T\right)$ for different thicknesses, all for a value of $L=100$. (b) Dependence of the inverse critical exponent $\beta^{*-1}$ on the thickness of the system. The data follow an exponential decay ( $D=20$ from Ref. [5]). 
critical effective exponent $\left(\beta^{*}(D)\right)$ just before the crossover begins. In this case it is also possible to detect a residual value for the magnetization as we approach the critical point [16], due to the finite value of $L$ used. The values $1 / \beta^{*}(D)$ for different thicknesses are shown in Fig. 3b. Again, we find a strong thickness dependence of $1 / \beta^{*}(D)$ on an exponential decay towards the three-dimensional value.

Once the exponents $\beta^{*}(D)$ and $\delta^{*}(D)$ are known, it is straightforward to calculate the exponent $\gamma^{*}(D)$, using the scaling equality

$$
\gamma=\beta(\delta-1) \text {. }
$$

This scaling relation has been checked for thin Ising films all over the crossover region by renormalization group analysis [4] and more recently by Monte Carlo simulations [15].

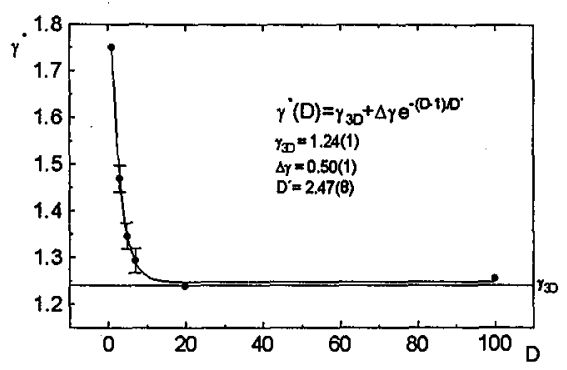

Fig. 4. Dependence of the critical exponent $\gamma^{*}$ calculated using the scaling relation $\gamma=\beta(\delta-1)$ on the thickness. The data follow an exponential decay.

The behavior of $\gamma^{*}(D)$ so obtained is shown in Fig. 4. Using Eq. (4) we find the behavior for $\gamma^{*}$ very similar to the one previously reported for the other effective exponents. That is, we find a strong thickness dependence and an exponential decay behavior towards the three-dimensional value.

\section{TABLE}

Critical temperature and critical exponents for various thicknesses $(D)$ in Ising thin films.

\begin{tabular}{c|c|c|c|c|c|c}
\hline \hline & $D=1$ & $D=3$ & $D=5$ & $D=7$ & $D=20$ & $D=L$ \\
\hline$T_{\mathrm{c}}$ & 2.2692 & 4.0649 & 4.3212 & 4.4011 & - & 4.5116 \\
$\delta^{*}$ & 15 & 11.9 & 9.03 & 7.57 & 5.13 & 4.8 \\
$\beta^{*}$ & 0.125 & 0.133 & 0.168 & 0.197 & 0.3 & 0.33 \\
$\gamma^{*}$ & 1.75 & 1.47 & 1.35 & 1.29 & 1.24 & 1.24
\end{tabular}

Table gives the values of the critical temperature and the critical exponents calculated for the thicknesses investigated, along with the values for the two-dimensional and three-dimensional cases. 


\section{Conclusions}

In conclusion, we have numerically characterized effective critical exponents for Ising plates of different thicknesses, using periodic boundary conditions. The transition of critical temperature and of maximum effective critical exponents from two-dimensional to three-dimensional values takes place in a relatively small thickness interval. The values of the effective critical exponents $\left(\beta^{*}, \delta^{*}\right.$, and $\left.\gamma^{*}\right)$ strongly depend on the thickness of the Ising lattice, in opposition to the true critical exponents, which are always the ones corresponding to the two-dimensional case. This $D$ dependence allows one to use these exponents to characterize the thickness of thin Ising films.

However, it is important to note that the effective exponents cannot be used to scale data near the critical point, since the universality class of these systems is always two-dimensional. But, it is not excluded that they are capable to scale the data in the region before the crossover takes place, at least for small values of $L$, using a properly chosen effective $T_{\mathrm{c}}$.

The scaling prediction for $T_{\mathrm{c}}(D)$ (Eq. (1)) has been shown to be fulfilled from $D=3$ up, rather than for thicknesses greater than 20 as is shown by Schilbe et al. [5].

We acknowledge to the organizers of the XXIII International School on Ferroelectrics Physics celebrated in Kudowa Zdrój (Poland), September 1998, the opportunity to participate in the School, where this paper was presented. Financial support from DGCyT through the grant PB96-0037 is gratefully acknowledged.

\section{References}

[1] K. Binder, D.P. Landau, Phys. Rev. Lett. 52, 318 (1984).

[2] C. Ruge, F. Wagner, Phys. Rev. B 52, 4209 (1995).

[3] T.W. Capehart, M.E. Fisher, Phys. Rev. B 13, 6021 (1976).

[4] D. O‘Connor, C.R. Stephens, Phys. Rev. Lett. 72, 506 (1994).

[5] P. Schilbe, S. Siebentritt, K.-H. Rieder, Phys. Lett. A 216, 20 (1996).

[6] W. Janke, K. Nather, Phys. Rev. B 48, 15807 (1993).

[7] R.H. Swendsen, J.-S. Wang, Phys. Rev. Lett. 58, 86 (1987); U. Wolf, Phys. Lett. $B$ 228, 379 (1989).

[8] W.H. Press, S.A. Teukolsky, W.T. Vetterling., B.P. Flannery, Numerical Recipes in Fortran, Cambridge University Press, Cambridge 1992.

[9] K. Binder, Thin Solid Films 20, 367 (1974).

[10] K. Binder, Z. Phys. B 43, 119 (1981).

[11] M.E. Fisher, in: Critical Phenomena, Proc. Int. School of Physics "Enrico Fermi", Varenna, Italy, Ed. M.S. Green, Course LI., Academic Press, New York 1971, p. 1.

[12] V. Privman, in: Finite Size Scaling and Numerical Simulation of Statistical Systems, Ed. V. Privman, World Scientific, Singapore 1990, p. 1.

[13] D.P. Landau, K. Binder, Phys. Rev. B 31, 5946 (1985).

[14] A.M. Ferrenberg, D.P. Landau, Phys. Rev. B 44, 5081 (1991).

[15] M.I. Marqués, J.A. Gonzalo, Eur. Phys. J. B 14, 317 (2000).

[16] M.I. Marqués, J.A. Gonzalo, Physica A 267, 165 (1999). 